\title{
Affective blindsight relies on low spatial frequencies
}

\author{
Nicolas Burra ${ }^{\mathrm{b}}$, Alexis Hervais-Adelman ${ }^{\mathrm{c}, \mathrm{d}}$, Alessia Celeghin ${ }^{\mathrm{e}, \mathrm{f}}$, Beatrice de Gelder ${ }^{g}$, \\ Alan J. Pegna ${ }^{\text {a,* }}$ \\ a School of Psychology, The University of Queensland, Saint Lucia, Brisbane Qld-4072, Australia \\ b Faculty of Psychology and Educational Science, University of Geneva, Geneva, Switzerland \\ c Neuroscience Center, University of Geneva, Switzerland \\ d Brain and Language Laboratory, University of Geneva Medical School, University of Geneva, CH-1211 Geneva, Switzerland \\ e Department of Medical and Clinical Psychology, and CoRPS - Center of Research on Psychology in Somatic diseases - Tilburg University, Tilburg, The Netherlands \\ ${ }^{\mathrm{f}}$ Department of Psychology, University of Turin, Turin, Italy \\ ${ }^{g}$ Brain and Emotion Laboratory, Maastricht University, Maastricht, The Netherlands
}

\section{A R T I C L E I N F O}

\section{Keywords:}

Affective blindsight

Amygdala

Facial expressions

Fear emotion

Spatial frequency

Hemianopia

\begin{abstract}
A B S T R A C T
The human brain can process facial expressions of emotions rapidly and without awareness. Several studies in patients with damage to their primary visual cortices have shown that they may be able to guess the emotional expression on a face despite their cortical blindness. This non-conscious processing, called affective blindsight, may arise through an intact subcortical visual route that leads from the superior colliculus to the pulvinar, and thence to the amygdala. This pathway is thought to process the crude visual information conveyed by the low spatial frequencies of the stimuli.

In order to investigate whether this is the case, we studied a patient (TN) with bilateral cortical blindness and affective blindsight. An fMRI paradigm was performed in which fearful and neutral expressions were presented using faces that were either unfiltered, or filtered to remove high or low spatial frequencies. Unfiltered fearful faces produced right amygdala activation although the patient was unaware of the presence of the stimuli. More importantly, the low spatial frequency components of fearful faces continued to produce right amygdala activity while the high spatial frequency components did not. Our findings thus confirm that the visual information present in the low spatial frequencies is sufficient to produce affective blindsight, further suggesting that its existence could rely on the subcortical colliculo-pulvino-amygdalar pathway.
\end{abstract}

\section{Introduction}

In 1973, Larry Weiskrantz coined the term "blindsight" to designate the astonishing ability that is sometimes observed in cortical blindness, whereby patients might detect the spatial location, or correctly guess certain basic characteristics of visual stimuli, such as shape, motion, or wavelength (Weiskrantz, 1986, 2010; Weiskrantz et al., 1974). Surprisingly, the study of one such blindsight patient (GY) revealed that blindsight could also occur for apparently more complex visual features including emotional facial expressions, pointing to the existence of an affective form of blindsight (de Gelder et al., 1999). The reliability of affective blindsight was further corroborated in a subsequent patient (TN) who had lost both visual hemifields following two consecutive bilateral strokes that rendered him cortically blind, and who also revealed an above-chance performance when guessing facial expressions, and who further showed right amygdala activation in response to these stimuli (Pegna et al., 2005).
One of the key questions arising from these observations is by what pathway the amygdala receives its visual information, particularly in cases such as TN, who showed a complete destruction of his primary visual cortices (i.e., a loss of both left and right V1). Several explanations have been put forward to account for these findings (reviewed in Celeghin et al. (2015)). One influential hypothesis (LeDoux, 1996, 2012) suggests that the amygdala is activated through a subcortical projection that bypasses cortical regions, and allows rapid, coarse processing of threatening stimuli (LeDoux, 1996), and possibly more generally, of stimuli that are essential for survival (LeDoux, 2012). A growing body of evidence points to the existence of such a subcortical pathway, which is thought to project from the retina to the superior colliculus and pulvinar, and thence to the amygdala (see discussions by Tamietto and de Gelder (2010), Diano et al. (2017), Tamietto and Morrone (2016)). Moreover, evidence of activation of this pathway for facial expressions has been obtained in healthy participants and in patients with cortical blindness (Morris et al., 1999). However, this

\footnotetext{
* Corresponding author.

E-mail address: a.pegna@uq.edu.au (A.J. Pegna).
} 
Table 1

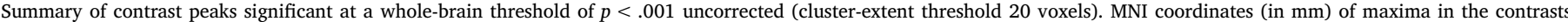

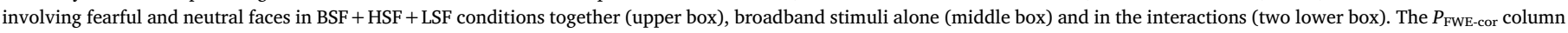
indicates familywise-error corrected probability values for contrasts reaching criteria for significance within the right amygdala ROI (ns: non-significant).

\begin{tabular}{|c|c|c|c|c|c|c|}
\hline Side & Area & $x$ & $y$ & $z$ & $t$ & $P_{\text {FWE-cor }}{ }^{\text {a }}$ \\
\hline \multicolumn{7}{|c|}{ Fearful > Neutral (collapsed across all spatial frequencies) } \\
\hline $\mathrm{R}$ & Anterior Cingulate Cortex & 6 & 46 & 10 & 4.30 & \\
\hline $\mathrm{R}$ & Posterior Cingulate Cortex & 12 & -48 & 24 & 3.92 & \\
\hline $\mathrm{R}$ & Middle Temporal Gyrus & 44 & -62 & 22 & 3.53 & \\
\hline $\mathrm{L}$ & Superior Medial Gyrus & -12 & 50 & 12 & 3.67 & \\
\hline $\mathrm{R}$ & Superior Frontal Gyrus & 22 & 34 & 32 & 3.69 & \\
\hline $\mathrm{R}$ & Thalamus & 6 & -4 & 2 & 3.75 & \\
\hline \multicolumn{7}{|c|}{ Fearful $>$ Neutral (Broadband) } \\
\hline $\mathrm{R}$ & Posterior Cingulate Cortex & 12 & -46 & 26 & 3.87 & \\
\hline $\mathrm{R}$ & Angular Gyrus & 42 & -60 & 24 & 4.07 & \\
\hline $\mathrm{R}$ & Precuneus & 2 & -48 & 54 & 3.64 & \\
\hline $\mathrm{R}$ & Superior Temporal Gyrus & 66 & -10 & 6 & 4.25 & \\
\hline $\mathrm{R}$ & Thalamus & 6 & -2 & 2 & 3.69 & \\
\hline $\mathrm{L}$ & Middle Temporal Gyrus & -48 & -36 & -14 & 4.06 & \\
\hline $\mathrm{L}$ & Precentral Gyrus & -36 & -12 & 44 & 4.26 & \\
\hline $\mathrm{R}$ & Middle Temporal Gyrus & 58 & -32 & -12 & 3.41 & \\
\hline $\mathrm{L}$ & Insula Lobe & -28 & 32 & 8 & 3.66 & \\
\hline $\mathrm{R}$ & Amygdala & 22 & -2 & -14 & 3.40 & .011 \\
\hline \multicolumn{7}{|c|}{ Spatial Frequencies X Facial expression Interaction (Fearful LSF > HSF) } \\
\hline $\mathrm{L}$ & Precentral Gyrus & -30 & -14 & 58 & 4.48 & \\
\hline $\mathrm{R}$ & Mid Orbital Gyrus & 10 & 38 & -8 & 4.75 & \\
\hline $\mathrm{R}$ & Middle Temporal Gyrus & 62 & -34 & 0 & 4.27 & \\
\hline $\mathrm{L}$ & Inferior Frontal Gyrus & -28 & 32 & -8 & 4.94 & \\
\hline $\mathrm{R}$ & Middle Cingulate Cortex & 16 & -44 & 36 & 4.33 & \\
\hline $\mathrm{R}$ & Superior Orbital Gyrus & 20 & 54 & -8 & 3.97 & \\
\hline $\mathrm{L}$ & Putamen & -20 & 16 & -8 & 4.13 & \\
\hline $\mathrm{R}$ & Amygdala* & 20 & -6 & -6 & 2.59 & ns \\
\hline \multicolumn{7}{|c|}{ Spatial Frequencies X Facial expression Interaction (Fearful LSF > Neutral LSF) } \\
\hline $\mathrm{R}$ & Inferior Parietal Lobule & 50 & -72 & 30 & 4.90 & \\
\hline $\mathrm{L}$ & Superior Orbital Gyrus & -16 & 30 & -12 & 3.38 & \\
\hline $\mathrm{R}$ & Superior Orbital Gyrus & 22 & 36 & -10 & 3.75 & \\
\hline $\mathrm{R}$ & Middle Occipital Gyrus & 38 & -88 & 18 & 4.48 & \\
\hline $\mathrm{R}$ & Inferior Temporal Gyrus & 50 & -66 & 0 & 3.36 & \\
\hline $\mathrm{R}$ & Amygdala & 20 & -6 & -6 & 4.07 & .021 \\
\hline
\end{tabular}

All Peaks $P<.001$ uncorrected (cluster-extent threshold $=20$ voxels).

$* P<.005$.

${ }^{\text {a }}$ Corrected for multiple comparisons within the right amygdala ROI.

hypothesis has been disputed by others who argue that the evidence for this retino-tectal pathway is insufficient, and that some forms of nonconscious processing in healthy as well as blindsight subjects would best be explained by geniculo-extrastriate projections (for reviews, see e.g., Pessoa and Adolphs, 2010; Leopold, 2012). This has led to a strong debate around the validity of the role of the subcortical pathway in emotional face processing and in affective blindsight.

One line of research exploring the subcortical pathway in emotion processing, hinges on the idea that it processes coarse visual information. The reasoning here is that if the colliculo-pulvinar route rapidly conveys crude information regarding the stimuli, then the low spatial frequency components of the emotional face should suffice to activate the amygdala (De Cesarei and Codispoti, 2013). In line with this suggestion, a number of studies have presented emotional faces filtered of their high or low spatial frequency spectrum and have looked into amygdala activity. Most notably, in one fMRI investigation of healthy participants, fearful and neutral faces were presented visually and the BOLD response was measured in order to explore activation differences in the amygdala and fusiform face area (Vuilleumier et al., 2003). Fearful and neutral faces were presented either unfiltered (broad spatial frequency -BSF-), or filtered to reveal only the Low Spatial Frequency (LSF), or the High Spatial Frequency (HSF). Convincingly, the results showed that amygdala activity arose for BSF and LSF faces, but not for HSF stimuli, arguing in favour of a subcortical pathway biased towards low spatial frequencies (Vuilleumier et al., 2003). Since this report, a number of other studies have highlighted the importance of LSF in emotional face processing (e.g., Bocanegra and Zeelenberg, 2009; Mermillod et al., 2009), yet the reliance of the subcortical pathway on LSF remains disputed (e.g., De Cesarei and Codispoti, 2013; Garrido, 2012; Garvert et al., 2014; McFadyen et al., 2017).

Until now, these reports have all studied healthy control participants in whom both subcortical and geniculo-striate pathways are intact and who therefore have both pathways available for use under the different viewing conditions. One approach that could shed more light on this question would be therefore to examine brain responses to LSF and HSF faces in brain-damaged individuals, whose geniculo-striate routes are injured, i.e., who suffer from cortical blindness. If amygdala activation can be produced with LSF rather than HSF in this situation, this would suggest that affective blindsight relies mainly on magnocellular input and would further strengthen the idea of a subcortical route conveying crude visual information.

To investigate this, we tested a well-known patient suffering from cortical blindness (TN), who is known to present affective blindsight, and in whom right amygdala activation was previously found in response to facial expressions (Pegna et al., 2005). The current paradigm addressed the issue of spatial frequency by measuring the BOLD response during the presentation of fearful and neutral faces that were spatially filtered to remove the high or low spatial frequency components.

We hypothesized that if the retino-tectal pathway processes primarily LSF, faces removed of their HSF components should continue to produce amygdala activation, while this would not be the case for faces 


\section{Neutral}

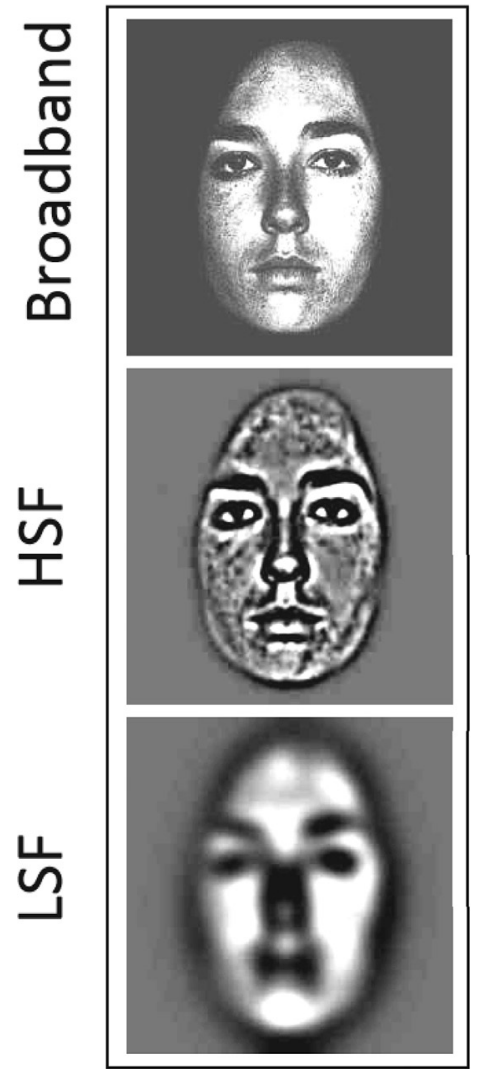

Fig. 1. Stimuli we composed of unfiltered (broadband) images of neutral and fearful faces (top row), as well as versions of the same photographs filtered to reveal only their high spatial frequencies (HSF; 2nd row) or low spatial frequencies (LSF; bottom row).

filtered for their low spatial frequencies.

\section{Methods and material}

\subsection{Case report}

Patient TN is a male physician who was 60 years of age at the time of testing. He suffered two consecutive strokes at the age of 52, the first in the left parieto-temporo-occipital cerebral area, causing right hemianopia, hemiplegia and transcortical sensory aphasia (the latter two symptoms receded rapidly). A second hemorrhage then occurred in the right occipital lobe producing a loss of the remaining (left) visual field, giving rise to complete cortical blindness.

Structural MRI shows that the lesion in the left hemisphere includes most of the occipital lobe, with minimal sparing of the medial ventral part of the inferior occipital gyrus and anterior part of the lingual gyrus. The lesion extends anteriorly to the middle part of the fusiform gyrus leaving the parahippocampal gyrus grossly intact. Laterally, the lesion extends to the medial inferior temporal gyrus. Dorsally, it reaches the superior parietal lobule and spares the ventral part of the precuneus. The right hemisphere lesion is smaller and includes most of the occipital lobe, with limited sparing of the medial part of the posterior lingual gyrus and medial part of precuneus. The anterior border stretches to the middle part of the fusiform gyrus and includes the posterior inferior temporal gyrus, but spares the parahippocampal gyrus (see Van den Stock et al. (2014), for a 3D reconstruction of the lesions). No anatomic input to striate areas could be detected in either left or right hemisphere using DTI (de Gelder et al., 2008).

\subsection{Stimuli}

Six different identities ( 3 male, 3 female) were used, similar to our first study of this patient (Pegna et al., 2005). This material consisted of fearful and neutral faces, obtained from the Ekman database. Presented on a black background, each picture, $512 * 512$ pixels, was then filtered using the parameters employed in previous paradigms (Burra et al., 2013; Vuilleumier et al., 2003). Using Image-J and a procedure suggested by other authors (Delplanque et al., 2007), these pictures were de-saturated and equalized for their facial luminance between conditions. This yielded three filtering conditions: normal (unfiltered) pictures, HSF pictures (filtered using a high-pass cut-off of $>32$ cycles/ image) and LSF pictures (filtered using a low-pass cut-off of $<6$ cycles/ image).

\section{3. fMRI procedure}

TN, lying in the MRI scanner, was simply requested to keep his eyes open during the experimental presentation. Stimuli were projected onto a screen situated at the end of the bore closest to the patient's head. A mirror placed on the head coil reflected projected images directly towards the centre of the patient's line of gaze when he looked straight ahead. After verifying the position of the display mirror (prior to inserting TN into the bore of the scanner), we monitored TN's gaze direction scrupulously during the recording session with an eye tracker camera, although we were unable to calibrate the eye tracker due to the impossibility of having him fixate.

We presented a total of 24 blocks of stimuli, each lasting $24 \mathrm{~s}$. Every block contained 6 items of the same condition presented for $2 \mathrm{~s}$ each (Neutral (N), Fearful (F), Neutral low frequencies (Neutral-LSF), Fearful low frequencies (Fearful-LSF), Neutral high frequencies (Neutral-HSF), Fearful high frequencies (Fearful-HSF)). These conditions were presented in a pseudo-random order. Every block was succeeded by a $24 \mathrm{~s}$ period of no stimulation. As a result, 4 blocks of each of the 6 conditions ( 2 emotions: F, N; 3 spatial filterings: BSF, LSF, HSF) were delivered, with each single picture presented once per block.

\subsection{MRI data acquisition}

Scanning took place in a 3T Siemens Trio MRI scanner, using a 12channel head-coil. 340 functional volumes were acquired in one single session, lasting approximately $7 \mathrm{~min}$ (T2*-weighted EPI, 32 sequential $3 \mathrm{~mm}$ slices, $15 \%$ interslice gap, $2.56 * 2.56 \mathrm{~mm}$ in-plane resolution, angled away from the eyes to prevent ghost-artefacts from aliasing of eye-movements, TA: $2 \mathrm{~s}$, TR: $2 \mathrm{~s}$, TE: $30 \mathrm{~ms}$ ). A high-resolution (.43 $\mathrm{mm} * .43 \mathrm{~mm} * 1 \mathrm{~mm}$ voxels) T1-weighted anatomical image was also acquired.

\section{5. fMRI preprocessing and analysis}

Analysis was carried out using the SPM 8 software package (http:// www.fil.ion.ucl.ac.uk/spm/), with the following steps (1) realignment: EPI volumes were realigned using a two-pass procedure. Images were initially realigned to the first volume, and a mean image was generated, to which the EPI volumes were then realigned; (2) coregistration of the structural volume to the mean EPI image and then (3) spatial smoothing using a Gaussian kernel of $8 \mathrm{~mm}$ at full-width half-maximum height.

In order to compare the patient's neuroanatomy with that of existing atlases, we normalized the T1 anatomical image to the standard MNI template included in SPM8, using a dedicated toolbox, i.e. the "clinical toolbox", which is robust in the face of lesions. The normalization parameters generated by this process were subsequently applied to the contrast images produced by the functional analysis. Activation coordinates are reported in MNI space and region identification was determined using the SPM Anatomy Toolbox (Eickhoff et al., 2005).

Nonetheless, it is important to note that all figures, beta values and 


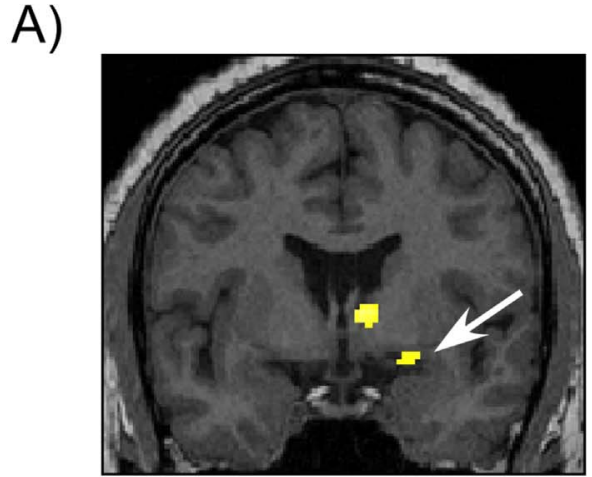

B)

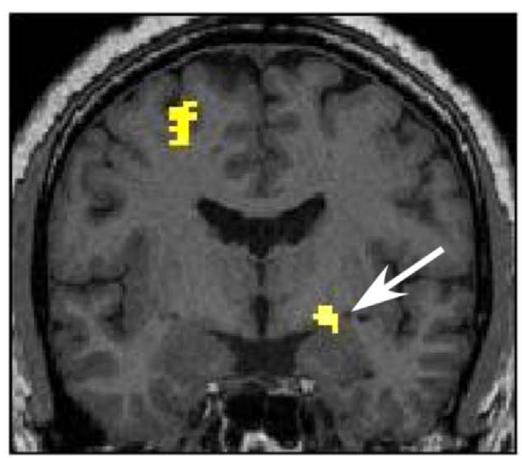

C)

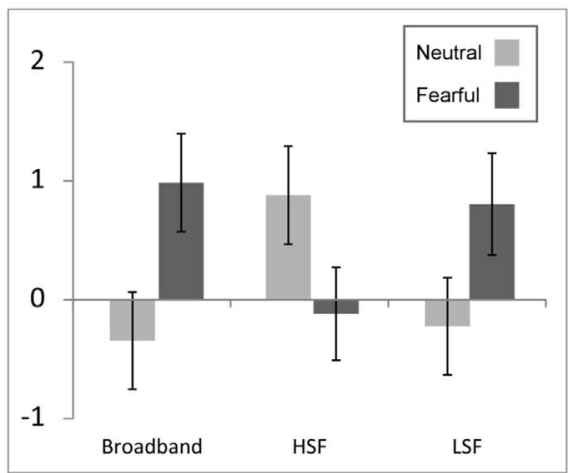

D)

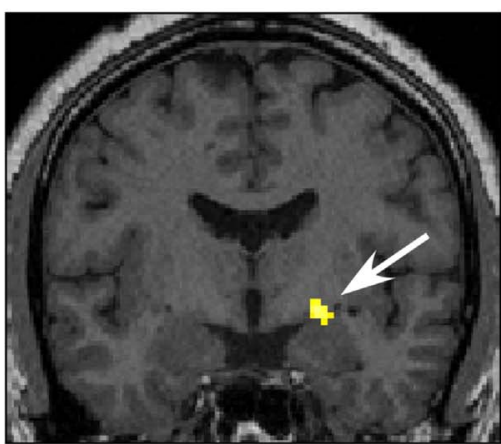

functional connectivity analyses that we report were performed on the data in TN's native space.

We used a general linear model (GLM) in which every scan was coded for condition. The BOLD response to each stimulus was modelled using the canonical hemodynamic response function (HRF). Six movement parameters ( $\mathrm{x}, \mathrm{y}$ and $\mathrm{z}$ translations and $\mathrm{x}, \mathrm{y}$ and $\mathrm{z}$ rotations) derived from the realignment step described above were included in the model. A high-pass filter (cutoff $128 \mathrm{~s}$ ) and AR1 correction for serial autocorrelation were applied.

All results reported in this paper reach a significance level of $P<.001$ uncorrected, unless otherwise stated, with a minimal extent of 20 voxels. It should be noted, however, that no contrasts yielded significant results at the $P<.05$ threshold (corrected for multiple comparison across the whole brain).

Due to our specific focus on the right amygdala we carried out a region of interest (ROI) analysis restricted to this structure. The right amygdala ROI was generated anatomically using the PickAtlas tool (Maldjian et al., 2003) in SPM8. We used the same parameters as in the whole brain analysis, but report only the corresponding corrected $P$ values on cluster levels if the family-wise error (FWE) probability was $<.05$ (see Table 1 ).
Fig. 2. BOLD response obtained during the experimental procedure with patient TN (superimposed on his anatomical scan). Amygdala activity (arrow) was observed in response to fearful faces, compared to neutral faces, both in the broadband (A) and the low spatial frequency conditions (D). The amygdala also emerged in the facial expression $\mathrm{X}$ spatial frequency interaction (B). In C, the average beta estimate in the right amygdala ( \pm S.E.M.) is illustrated across conditions (mean $\mathrm{x}, \mathrm{y}, \mathrm{z}=20,-6,-6$; cluster extent threshold 20 voxels at $P<.001$ ); from left to right, bars show responses for unfiltered stimuli (Broadband), then high spatial frequency faces (HSF) and finally low spatial frequency faces (LSF). Light grey bars indicate responses for neutral faces and dark grey bars for fearful faces. 


\subsubsection{Interaction [Fearful HSF $<$ Neutral HSF] - [Fearful LSF $>$ Neutral} LSF]

Using a contrast similar to that in Vuilleumier et al. (2003), we explored the impact of the interaction effect in filtered pictures. As illustrated in Fig. 2, we found a significant effect in the amygdala (Fig. 2B) [MNI: 20, -6, -6] for this interaction contrast (Fig. 2C) in the amygdala $\mathrm{ROI}\left(P_{\mathrm{FWE}-\mathrm{cor}}=.022\right)$.

\subsubsection{Fearful $L S F>$ neutral $L S F$}

A post-hoc analysis revealed that the interaction was explained mainly by a larger activation for fearful faces compared to neutral ones in LSF conditions. This contrast was significant in the amygdala ROI $\left(P_{\text {FWE-cor }}=.021\right)$.

\subsubsection{Fearful $L S F>$ fearful $H S F$}

When directly comparing fearful expressions in the LSF and HSF conditions, we found a stronger activation for fearful LSF than fearful HSF expressions in the same amygdala region, highlighted by the previous contrast [MNI: $20,-6,-6]$ at $P<.005$. However, this effect did not meet the criterion for significance when correcting for multiple comparisons in the amygdala ROI $\left(P_{\text {FWE-cor }}=.404\right)$.

\section{Discussion}

The current fMRI investigation of patient TN, who suffered bilateral destruction of his primary visual cortices, revealed the presence of significantly elevated right amygdala activation in response to emotional faces, as compared to neutral faces. Most noteworthy, our study revealed for the first time in a patient with blindsight and complete cortical blindness that the low spatial frequency $(<6 \mathrm{~Hz})$ information contained in the fearful faces was sufficient to elicit the amygdala response. This suggests the functional relevance of an alternative, extrageniculo-striate pathway, conveying coarse visual information without implicating V1. As noted above, one of the likely routes for this may be a subcortical, predominantly magnocellular, pathway conveying information to the superior colliculus and pulvinar, then projecting to the amygdala, which would allow the occurrence of nonconscious processing and affective blindsight.

Evidence consistent with the role of this subcortical pathway in nonconscious emotion processing emerges from a number of different observations (see Diano et al. (2017) for a review). Brain imaging studies of healthy human participants, revealed amygdala responses to fearful faces over two decades ago (Morris et al., 1996), while this activation was later found to co-vary with that in the superior colliculus and the pulvinar (Calder et al., 1996; Morris et al., 1999). On the other hand, lesion studies in humans have shown that damage to the amygdala (Adolphs et al., 1994; Calder et al., 1996) or the pulvinar (Ward et al., 2007) impair the recognition of facial expressions, in particular fear, thus supporting their role in emotional face processing. More recent anatomical findings have added evidence for the existence of this circuit by showing connections between these structures in humans. Indeed, using tractography, Tamietto et al. (2012) and Rafal et al. (2015) demonstrated the presence of fibres connecting the superior colliculus with the amygdala via the pulvinar, in a group of healthy controls (as well as in monkeys, in the latter study). Additionally, Tamietto et al. (2012) included a patient with unilateral V1 damage (GY) and blindsight and found an increase in the density of connections of the subcortical pathway, compared with the intact hemisphere suggesting that the subcortical pathway may develop after brain damage and could well contribute to blindsight. A future tractographic exploration of this anatomical pathway in TN would therefore appear a necessary contribution to confirm this point.

Electrophysiological data from monkeys have provided further support for the implication of the superior colliculus and pulvinar in this putative subcortical pathway. Indeed, electrophysiological recordings in the macaque have revealed the existence of neurones sensitive to faces in both structures. Nguyen et al. (2013) recorded single unit activity in the macaque pulvinar and found that the activity in the initial $50 \mathrm{~ms}$ period differentiated face-like stimuli from other categories. Even more compellingly, Maior et al. (2010) obtained measures from 184 pulvinar neurons in 2 macaques while they viewed human faces exhibiting sad, angry, happy, surprised and neutral expressions. Of the cells found to be visually responsive, almost half were sensitive to human facial expressions, confirming that facial expressions are represented in these structures. Likewise, single unit measures in the monkey superior colliculus have also identified neurones that respond to face, or face-like stimuli (Nguyen et al., 2014). Of particularly relevance, this response was not altered when higher spatial frequencies were filtered out (unfortunately, here the effects of low frequency removal were not examined). Thus, each relay of the retino-tectal route to the amygdala appears to possess the ability to process faces, confirming that complex stimuli can be processed along this path.

As mentioned above, this subcortical route is thought to rely on the low spatial frequencies of the emotional stimuli, for which evidence was initially provided in a study by Vuilleumier et al. (2003). These investigators carried out an fMRI study while healthy participants viewed spatially-filtered fearful and neutral faces, removed of their high or low frequency content. As expected, activity for fearful faces was observed in the amygdala, in conjunction with the superior colliculus and pulvinar. More importantly, this effect was reported for unfiltered faces and for LSF stimuli, while the HSF stimuli failed to produce this result (Vuilleumier et al., 2003). Similarly, intracranial recordings carried out in patients during their work-up for surgical alleviation of their epileptic seizures revealed early-latency amygdala activation for fearful faces that remained present for stimuli when the high spatial frequencies, but not the low spatial frequencies, were removed (Mendez-Bertolo et al., 2016). Together with our present findings, the facts support the notion of a subcortical pathway processing low spatial frequencies information regarding emotional faces. The primacy of LSF information in the subcortical pathway appear to derive from the predominance of magnocellular projections to the superior colliculus (e.g., Markus et al., 2009), which favours speed over detail. Indeed, the low spatial frequency components of fearful faces have been reported to produce more rapid responses than the high spatial frequency components (Vlamings et al., 2009). However, a series of recent magneto-encephalography (MEG) investigations have addressed the question of the different spatial frequencies processed by the colliculopulvinar route for the different emotional faces and have reached different conclusion. Garvert et al. (2014) used MEG and dynamic causal modelling (DCM) to examine which model of the cortical or dual (subcortical and cortical) routes best explained the pattern of activation, in a task in which happy, fearful and neutral faces were presented. Essentially, two groups of models were tested, one in which the amygdala would receive information via the geniculo-striate route alone, and one in which information would also be conveyed to the amygdala via the pulvinar (with additional variants including different feedback loops). MEG activation patterns were best explained by the dual model, particularly during the earlier processing periods. However, the results did not show any differences across emotions, leading the author to conclude that the subcortical path is not fear-specific, and is therefore independent of emotion, echoing other observations, such as those with our current patient TN (Pegna et al., 2005). Furthermore, McFadyen et al. (2017) recently replicated this study, this time filtering out the low and high spatial frequency of the emotional faces. Their observations again showed a superior fit for the model that included both a geniculo-striate path to the amygdala and a subcortical path with pulvino-amygdalar (and pulvino-striate) projections. In addition to the absence of an emotion-specific effect, the study also failed to find any effect of spatial frequency. The existence of a subcortical, colliculoamygdalar pathway was therefore confirmed in these findings, but the reliance on LSF was questioned, in contrast to our current observations. In the midst of the existing controversy regarding the subcortical route 
and its dependence on LSF, our investigation of patient TN is revealing. TN's primary visual cortices are completely destroyed and previous investigations showed a lack of any responsive V1 tissue, or of any observable fibres connecting V1 (de Gelder et al., 2008). Despite his brain damage, he presents affective blindsight and shows amygdala activation for unfiltered and high-pass filtered fearful faces, demonstrating that the coarse visual features of the facial expressions continue to be processed.

These findings convincingly corroborate the hypothesis of a crude, subcortical colliculo-pulvinar pathway to the amygdala for emotional stimuli, additionally illustrating the importance of blindsight in the understanding of normal and pathological brain functions.

\section{Acknowledgments}

This investigation was supported by the Swiss National Science Foundation for Scientific Research (Grant no. \#320030-144187, P2GEP1_151949, PDFMP1_129459 and the authors declare no competing financial interests.

\section{References}

Adolphs, R., Tranel, D., Damasio, H., Damasio, A., 1994. Impaired recognition of emotion in facial expressions following bilateral damage to the human amygdala. Nature 372 (6507), 669-672. http://dx.doi.org/10.1038/372669a0.

Bocanegra, B.R., Zeelenberg, R., 2009. Emotion improves and impairs early vision. Psychol. Sci. 20 (6), 707-713. http://dx.doi.org/10.1111/j.1467-9280.2009. 02354.x.

Burra, N., Hervais-Adelman, A., Kerzel, D., Tamietto, M., de Gelder, B., Pegna, A.J., 2013. Amygdala activation for eye contact despite complete cortical blindness. J. Neurosci.: Off. J. Soc. Neurosci. 33 (25), 10483-10489. http://dx.doi.org/10.1523/ JNEUROSCI.3994-12.2013.

Calder, A.J., Young, A.W., Rowland, D., Perrett, D.I., Hodges, J.R., Etcoff, N.L., 1996. Facial emotion recognition after bilateral amygdala damage: differentially severe impairment of fear (Doi). Cogn. Neuropsychol. 13 (5), 699-745. http://dx.doi.org/ 10.1080/026432996381890.

Celeghin, A., de Gelder, B., Tamietto, M., 2015. From affective blindsight to emotional consciousness. Conscious. Cogn. 36, 414-425. http://dx.doi.org/10.1016/j.concog. 2015.05.007.

De Cesarei, A., Codispoti, M., 2013. Spatial frequencies and emotional perception. Rev. Neurosci. 24 (1), 89-104. http://dx doi.org/10.1515/revneuro-2012-0053.

de Gelder, B., Tamietto, M., van Boxtel, G., Goebel, R., Sahraie, A., van den Stock, J., Pegna, A., 2008. Intact navigation skills after bilateral loss of striate cortex. Curr. Biol.: CB 18 (24), R1128-R1129. http://dx.doi.org/10.1016/j.cub.2008.11.002.

de Gelder, B., Vroomen, J., Pourtois, G., Weiskrantz, L., 1999. Non-conscious recognition of affect in the absence of striate cortex. Neuroreport 10 (18), 3759-3763.

Delplanque, S., N'Diaye, K., Scherer, K., Grandjean, D., 2007. Spatial frequencies or emotional effects?? A systematic measure of spatial frequencies for IAPS pictures by a discrete wavelet analysis. J. Neurosci. Methods 165 (1), 144-150. http://dx.doi.org/ 10.1016/j.jneumeth.2007.05.030.

Diano, M., Tamietto, M., Celeghin, A., Weiskrantz, L., Tatu, M.K., Bagnis, A., Costa, T., 2017. Dynamic changes in amygdala psychophysiological connectivity reveal distinct neural networks for facial expressions of basic emotions. Sci. Rep. 7, 45260. http:// dx.doi.org/10.1038/srep45260.

Eickhoff, S.B., Stephan, K.E., Mohlberg, H., Grefkes, C., Fink, G.R., Amunts, K., Zilles, K., 2005. A new SPM toolbox for combining probabilistic cytoarchitectonic maps and functional imaging data. NeuroImage 25 (4), 1325-1335. http://dx.doi.org/10. 1016/j.neuroimage.2004.12.034

Garrido, M.I., 2012. Brain connectivity: the feel of blindsight. Curr. Biol.: CB 22 (15), R599-R600. http://dx.doi.org/10.1016/j.cub.2012.06.012.

Garvert, M.M., Friston, K.J., Dolan, R.J., Garrido, M.I., 2014. Subcortical amygdala pathways enable rapid face processing. NeuroImage 102 (Pt 2), 309-316. http://dx. doi.org/10.1016/j.neuroimage.2014.07.047.

GUY M MCKHANN, I.I., 2004. Non-invasive mapping of connections between human thalamus and cortex using diffusion imaging. Neurosurgery 54 (1).

LeDoux, J., 1996. Emotional networks and motor control: a fearful view. Prog. Brain Res.
107, 437-446.

LeDoux, J., 2012. Rethinking the emotional brain. Neuron 73 (4), 653-676. http://dx.doi. org/10.1016/j.neuron.2012.02.004.

Leopold, D.A., 2012. Primary visual cortex: awareness and blindsight. Annu. Rev. Neurosci. 35, 91-109. http://dx.doi.org/10.1146/annurev-neuro-062111-150356.

Maior, R.S., Hori, E., Tomaz, C., Ono, T., Nishijo, H., 2010. The monkey pulvinar neurons differentially respond to emotional expressions of human faces. Behav. Brain Res. 215 (1), 129-135. http://dx.doi.org/10.1016/j.bbr.2010.07.009.

Maldjian, J.A., Laurienti, P.J., Kraft, R.A., Burdette, J.H., 2003. An automated method for neuroanatomic and cytoarchitectonic atlas-based interrogation of fMRI data sets. NeuroImage 19 (3), 1233-1239.

Markus, Z., Berenyi, A., Paroczy, Z., Wypych, M., Waleszczyk, W.J., Benedek, G., Nagy, A., 2009. Spatial and temporal visual properties of the neurons in the intermediate layers of the superior colliculus. Neurosci. Lett. 454 (1), 76-80. http://dx.doi.org/10. 1016/j.neulet.2009.02.063.

McFadyen, J., Mermillod, M., Mattingley, J.B., Halasz, V., Garrido, M.I., 2017. A rapid subcortical amygdala route for faces irrespective of spatial frequency and emotion. J. Neurosci.: Off. J. Soc. Neurosci. 37 (14), 3864-3874. http://dx.doi.org/10.1523/ JNEUROSCI.3525-16.2017.

Mendez-Bertolo, C., Moratti, S., Toledano, R., Lopez-Sosa, F., Martinez-Alvarez, R., Mah, Y.H., Strange, B.A., 2016. A fast pathway for fear in human amygdala. Nat. Neurosci. 19 (8), 1041-1049. http://dx.doi.org/10.1038/nn.4324.

Mermillod, M., Vuilleumier, P., Peyrin, C., Alleysson, D., Marendaz, C., 2009. The importance of low spatial frequency information for recognising fearful facial expressions. Connect. Sci. 21 (1), 75-83 (doi:Pii 901821912 10.1080/ 09540090802213974).

Morris, J.S., Frith, C.D., Perrett, D.I., Rowland, D., Young, A.W., Calder, A.J., Dolan, R.J., 1996. A differential neural response in the human amygdala to fearful and happy facial expressions. Nature 383 (6603), 812-815. http://dx.doi.org/10.1038/ $383812 \mathrm{a} 0$.

Morris, J.S., Ohman, A., Dolan, R.J., 1999. A subcortical pathway to the right amygdala mediating "unseen" fear. Proc. Natl. Acad. Sci. USA 96 (4), 1680-1685.

Nguyen, M.N., Hori, E., Matsumoto, J., Tran, A.H., Ono, T., Nishijo, H., 2013. Neuronal responses to face-like stimuli in the monkey pulvinar. Eur. J. Neurosci. 37 (1), 35-51. http://dx.doi.org/10.1111/ejn.12020.

Nguyen, M.N., Matsumoto, J., Hori, E., Maior, R.S., Tomaz, C., Tran, A.H., Nishijo, H., 2014. Neuronal responses to face-like and facial stimuli in the monkey superior colliculus. Front. behav. neurosci. 8.

Pegna, A.J., Khateb, A., Lazeyras, F., Seghier, M.L., 2005. Discriminating emotional faces without primary visual cortices involves the right amygdala. Nat. Neurosci. 8 (1), 24-25. http://dx.doi.org/10.1038/nn1364.

Pessoa, L., Adolphs, R., 2010. Emotion processing and the amygdala: from a 'low road' to 'many roads' of evaluating biological significance. Nat. Rev. 11 (11), 773-783. http:// dx.doi.org/10.1038/nrn2920.

Rafal, R.D., Koller, K., Bultitude, J.H., Mullins, P., Ward, R., Mitchell, A.S., Bell, A.H., 2015. Connectivity between the superior colliculus and the amygdala in humans and macaque monkeys: virtual dissection with probabilistic DTI tractography. J. Neurophysiol. 114 (3), 1947-1962. http://dx.doi.org/10.1152/jn.01016.2014.

Tamietto, M., de Gelder, B., 2010. Neural bases of the non-conscious perception of emotional signals. Nat. Rev. 11 (10), 697-709. http://dx.doi.org/10.1038/nrn2889.

Tamietto, M., Morrone, M.C., 2016. Visual plasticity: blindsight bridges anatomy and function in the visual system. Curr. Biol.: CB 26 (2), R70-R73. http://dx.doi.org/10. 1016/j.cub.2015.11.026.

Tamietto, M., Pullens, P., de Gelder, B., Weiskrantz, L., Goebel, R., 2012. Subcortical connections to human amygdala and changes following destruction of the visual cortex. Curr. Biol.: CB 22 (15), 1449-1455. http://dx.doi.org/10.1016/j.cub.2012. 06.006 .

Van den Stock, J., Tamietto, M., Zhan, M., Heinecke, A., Hervais-Adelman, A., Legrand, L.B., de Gelder, B., 2014. Neural correlates of body and face perception following bilateral destruction of the primary visual cortices. Front. behav. neurosci. 8 .

Vlamings, P.H., Goffaux, V., Kemner, C., 2009. Is the early modulation of brain activity by fearful facial expressions primarily mediated by coarse low spatial frequency information?? J. Vis. 9 (5) (12-12).

Vuilleumier, P., Armony, J.L., Driver, J., Dolan, R.J., 2003. Distinct spatial frequency sensitivities for processing faces and emotional expressions. Nat. Neurosci. 6 (6), 624-631. http://dx.doi.org/10.1038/nn1057.

Ward, R., Calder, A.J., Parker, M., Arend, I., 2007. Emotion recognition following human pulvinar damage. Neuropsychologia 45 (8), 1973-1978. http://dx.doi.org/10.1016/ j.neuropsychologia.2006.09.017.

Weiskrantz, L., 1986. Blindsight: a case study and implications.

Weiskrantz, L., 2010. Blindsight in hindsight. Psychologist 23 (4), 356-358.

Weiskrantz, L., Warrington, E.K., Sanders, M.D., Marshall, J., 1974. Visual capacity in the hemianopic field following a restricted occipital ablation. Brain 97 (4), 709-728. 